\title{
Kallikrein gene downregulation in breast cancer
}

\author{
GM Yousef ',2, GM Yacoub ${ }^{3}$, M-E Polymeris ${ }^{2}$, C Popalis'², A Soosaipillai' and EP Diamandis ${ }^{*, 1,2}$ \\ 'Department of Pathology and Laboratory Medicine, Mount Sinai Hospital, Toronto, Ontario, Canada; ${ }^{2}$ Department of Laboratory Medicine and \\ Pathobiology, University of Toronto, Toronto, Ontario, Canada; ${ }^{3}$ University of Virginia School of Medicine, Roanoke-Salem Internal Medicine Program, \\ Roanoke, VA 24033, USA
}

\begin{abstract}
Recent evidence suggests that many members of the human kallikrein gene family are differentially regulated in breast cancer and other endocrine-related malignancies. In this study, we utilised the serial analysis of gene expression (SAGE) and expressed sequence tag (EST) databases of the Cancer Genome Anatomy Project (CGAP) to perform in silico analyses of the expression pattern of the I 5 human kallikrein genes in normal and cancerous breast tissues and cell lines using different analytical tools such as Virtual Northern blotting, Digital Differential Display and X-profiler. Our results indicate that at least four kallikrein genes $(K L K 5,6,8,10)$ are downregulated in breast cancer. Probing eight normal and 24 breast cancer SAGE libraries with gene-specific tags for each of the above kallikreins indicated moderate-to-high expression densities in normal breast (27-319 tags per million; tpm, in two to five out of eight libraries), compared to no or low expression (0 - 34 tpm in zero to two libraries out of 24) in breast cancer. These data were verified by screening the EST databases, where all mRNA clones isolated for these genes, except for one in each, were from normal breast libraries, with no clones detected from breast cancer tissues or cell lines (with the exception of KLK8). X-profiler comparison of two pools of normal and breast cancer libraries further verified the presence of significant downregulation of expression levels of 4 of the kallikreins genes (KLK5, 6, 10, 12). We experimentally verified the downregulation of these four kallikreins $(K L K 5,6,8,10$ and 12) by RT - PCR analysis.

British Journal of Cancer (2004) 90, 167- 172. doi: I0.1038/sj.bjc.660 I45 I www.bjcancer.com
\end{abstract}

(C) 2004 Cancer Research UK

Cancer is fundamentally a disease of the genome. A key component in understanding the nature of cancer is the identification of those genes that are directly involved in or are associated with malignancy. In the past two decades, much progress has been made in identifying genes that play direct roles in the development of cancer. The Cancer Genome Anatomy Project (CGAP) aims to catalog all genes expressed during cancer development. Knowledge of these genes can then be utilised to understand the pathogenesis of cancer, to define its molecular signatures and to develop biomarkers for early detection and targets for intervention (Strausberg, 2001).

The Cancer Genome Anatomy Project focused on a strategy for gene identification based on isolation of mRNAs from tissues and generation of libraries from these transcripts. This library of genes, expressed in normal and cancer cells, has been developed through the application of two approaches; the expressed sequence tag (EST) (Adams et $a l, 1993$ ) and the serial analysis of gene expression (SAGE) (Velculescu et al, 1995). The SAGE method is similar to the EST approach, in that it provides a sequence tag for a portion of a cDNA. In this approach, the tagged sequences are generally quite short $(10-14 \mathrm{bp})$ and the individual tags are annealed to generate DNA molecules carrying many individual tags. Therefore, each DNA sequencing read generates tags for multiple transcripts. This method has been shown to quantitatively

\footnotetext{
*Correspondence: Dr EP Diamandis, Mount Sinai Hospital, Department of Pathology and Laboratory Medicine, 600 University Avenue, Toronto, Ontario Canada M5G IX5; E-Mail: ediamandis@mtsinai.on.ca Received 20 May 2003; revised 17 September 2003; accepted 6 October 2003
}

assess transcript levels and is more efficient than traditional EST sequencing. Thus, the opportunity to identify rarely expressed transcripts is increased in the SAGE approach.

Kallikreins are a family of 15 genes clustered together on chromosome 19 (Yousef and Diamandis, 2001, 2003) and encode for serine protease enzymes (Clements et al, 2001; Yousef and Diamandis, 2002b). Prostate-specific antigen (PSA, hK3), a member of this family, is an established tumour marker for prostate cancer (Barry, 2001). Prostate-specific antigen SA was recently found to be expressed in the female breast and might have a prognostic value in breast cancer (Black and Diamandis, 2000). Furthermore, other kallikreins were also found to have prognostic value in breast and other endocrine malignancies (Liu et al, 1996; Tanimoto et al, 1999; Yousef et al, 2000a; Benson et al, 2002; Diamandis and Yousef, 2002; Yousef and Diamandis, 2002a, 2003; Diamandis et al, 2003; Luo et al, 2003).

In this study, we used an in silico analysis approach to examine kallikrein gene expression in normal and cancerous breast tissues and cell lines. We provide evidence that at least four kallikreins are downregulated in breast cancer, and experimentally verified the downregulation of of these kallikreins (KLK5 and KLK12) by RT - PCR.

\section{MATERIALS AND METHODS}

\section{Serial Analysis of gene expression (SAGE)}

All publicly available SAGE data until August 2003 were used for analysis of kallikrein gene expression. We obtained a reliable mapping of UniGene (http://www.ncbi.nlm.nih.gov/UniGene/) 
groups to both NlaIII and Sau3A tags from the Serial Analysis of Gene Expression Tag to Gene Mapping (SAGEmap) search tool available through the NCBI web site (http://www.ncbi.nlm.nih.gov/). Each UniGene group consists of all GenBank sequences representing the same human gene. Hereafter, each such group is referred to as a 'gene'. Tags mapping to more than one kallikrein were excluded.

\section{Virtual Northern blots (VNB)}

The mRNA sequences of the 15 human kallikrein genes were used to identify unique sequence tags of UniGene clusters for each kallikrein (the GenBank reference sequences were used). Two restriction digestion enzymes were used (NlaIII, Sau3A). These sequence tags were then used to determine the levels of expression of different kallikreins in eight normal (six tissues and two cell lines) and 24 breast cancer (12 tissues and 12 cell lines) libraries. Detailed information of these libraries is available from the website of CGAP (http://www.ncbi.nlm.nih.gov/ncicgap/). Analyses were carried out by comparing the proportion of libraries of each type (normal $v s$ cancer) that show the expression of each tag in addition to the average expression densities in these libraries. mRNA sequences from the Human Genome Project were used as reference sequences. If more than one tag of the same gene appears in the same library, we only included the one with the highest expression (maximum tpm); the other tag was excluded to avoid inaccurate estimation of expression. Expression levels are displayed as blots with different densities and corrected as tpm to facilitate comparison. Table 1 shows the tags and UniGene clusters used for each gene to probe the GCAP libraries.

\section{Expressed sequence tag (EST) analysis}

The full-length mRNA sequence of each kallikrein was compared against the human EST databases of the NCBI. At the time of the study, these databases included seven normal and 14 breast cancer libraries. Expression was calculated for each kallikrein as the number of positive libraries out of the total in each tissue type, in addition to the total number of clones detected in each type.

\section{$\mathrm{X}$-profiler analysis}

$\mathrm{X}$-profiler analyses of kallikrein gene expression were performed by comparing normal and breast cancer libraries available in the SAGE databases. As expression levels of various kallikreins might

Table I Gene-specific SAGE tags used to probe different libraries of the CGAP databases

\begin{tabular}{|c|c|c|c|}
\hline Kallikrein & Restriction enzyme & SAGE tags & Unigene cluster \\
\hline \multirow[t]{4}{*}{$K L K 3$} & Nlalll & GGATGGGGAT & Hs. I71995 \\
\hline & & GTGACACAGC & \\
\hline & Sau3A & CACACTGAGA & Hs. 171995 \\
\hline & & ACGCTITTGT & \\
\hline \multirow[t]{2}{*}{$K L K 5$} & Nlalll & TCTCCTGGAC & Hs.509I5 \\
\hline & Sau3A & CAGGAAACCA & Hs.509I5 \\
\hline \multirow[t]{2}{*}{$K L K 6$} & Nlalll & CACTCAATAA & Hs.7936I \\
\hline & Sau3A & CAAAAAACCA & Hs.7936I \\
\hline \multirow[t]{2}{*}{$K L K 8$} & Nlalll & GTCTGTGCAG & Hs. 104570 \\
\hline & Sau3A & ТСССТТААТА & Hs. 104570 \\
\hline \multirow[t]{2}{*}{ KLKIO } & Nlalll & TAAGGCTTAA & Hs.69423 \\
\hline & Sau3A & CAGATGCCCA & Hs.69423 \\
\hline \multirow[t]{2}{*}{$K L K I I$} & Nlalll & GTGTGTGCCA & Hs.5777I \\
\hline & Sau3A & CAGGAGACGA & Hs.5777I \\
\hline \multirow[t]{2}{*}{$K L K / 2$} & Nlalll & AGGAACAACT & Hs. I 59679 \\
\hline & Sau3A & ATGAGGAACA & Hs. I 59679 \\
\hline
\end{tabular}

Only kallikreins that showed differential expression are shown in this table. be different from one cell line to another and in different types of breast cancer, we compared a pool of eight normal breast libraries (from tissues and cell lines) against 24 breast cancer libraries from tissues and cell lines. The X-profiler cut-off value was set at twofold difference.

\section{Digital differential display (DDD) analysis}

The DDD search engine (Strausberg et al, 2001) was used to compare EST expression in normal and cancer libraries. The databases at the time of analysis included seven normal and 14 breast cancer libraries. Libraries with less than 25 clones were excluded from the analysis.

\section{Normal and malignant breast tissues}

Normal breast tissues were obtained from four women undergoing reduction mammoplasties. Breast tumour tissues were obtained from 14 female patients at participating hospitals of the Ontario Provincial Steroid Hormone Receptor Program. The normal and tumour tissues were immediately frozen in liquid nitrogen after surgical resection and stored in this manner until extracted. The tissues were pulverised with a hammer under liquid nitrogen and RNA was extracted as described below. Our protocols were approved by the Institutional Review Board of the University of Toronto.

\section{Reverse transcriptase polymerase chain reaction (RT - PCR)}

Total RNA was extracted from the cell lines or tissues using Trizol reagent (Gibco BRL) following the manufacturer's instructions. RNA concentration was determined spectrophotometrically. Total RNA $(2 \mu \mathrm{g})$ was reverse-transcribed into first-strand cDNA using the Superscript ${ }^{\mathrm{TM}}$ preamplification system (Gibco, BRL, Gaithersburg, MD). The final volume was $20 \mu \mathrm{l}$. Two gene-specific primers were designed for each kallikrein (Table 2), and PCR was carried out in a reaction mixture containing $1 \mu \mathrm{l}$ of $\mathrm{cDNA}, 10 \mathrm{mM}$ Tris- $\mathrm{HCl}$ (pH 8.3), $50 \mathrm{~mm} \mathrm{KCl,} 1.5 \mathrm{~mm} \mathrm{MgCl}_{2}, 200 \mu \mathrm{M}$ dNTPs (deoxynucleoside triphosphates), $150 \mathrm{ng}$ of primers and $2.5 \mathrm{U}$ of AmpliTaq Gold DNA polymerase (Roche Molecular Systems, Branchburg, NJ, USA) on a Perkin-Elmer 9600 thermal cycler. The cycling conditions were $94^{\circ} \mathrm{C}$ for $15 \mathrm{~min}$ followed by 40 cycles of $94^{\circ} \mathrm{C}$ for $30 \mathrm{~s}$, annealing for $1 \mathrm{~min}$ (Table 2) and a final extension step at $63^{\circ} \mathrm{C}$ for $10 \mathrm{~min}$. Equal amounts of PCR products were

Table 2 Primers and conditions used for PCR analysis

\begin{tabular}{|c|c|c|c|c|}
\hline Gene & Sequence $^{\mathbf{a}}$ & $\begin{array}{l}\text { Product } \\
\text { length }^{b}\end{array}$ & $T_{a}^{c}$ & $\begin{array}{l}\text { Number } \\
\text { of cycles }\end{array}$ \\
\hline KLK5 & $\begin{array}{l}\text { GTCACCAGTTATGAATCTGGGC } \\
\text { GGCGCAGAACATGGTGTCATC }\end{array}$ & 328 & 60 & 35 \\
\hline KLK6 & $\begin{array}{l}\text { GAAGCTGATGGTGGTGCTGAGTCTG } \\
\text { GTCAGGGAAATCACCATCTGCTGTC }\end{array}$ & 454 & 60 & 35 \\
\hline KLK8 & $\begin{array}{l}\text { GCCTTGTTCCAGGGCCAGC } \\
\text { GCATCCTCACACTTCTTCTGGG }\end{array}$ & 416 & 65 & 35 \\
\hline$K L K I O$ & $\begin{array}{l}\text { GGAAACAAGCCACTGTGGGC } \\
\text { GAGGATGCCTTGGAGGGTCTC }\end{array}$ & 468 & 60 & 35 \\
\hline$K L K / 2$ & $\begin{array}{l}\text { GAGCAGATCCGGCACAGCGG } \\
\text { TCTTGTCCACAGGGCCCCACAG } \\
\text { GGCAGGGCGCAGCGCTCC }\end{array}$ & 425 & 69 & 35 \\
\hline$K L K / 5$ & $\begin{array}{l}\text { CTACGGACCACGTCTCGGGTC } \\
\text { GACACCAGGCTTGGTGGTGTTG }\end{array}$ & 459 & 65 & 35 \\
\hline$\beta$-actin & $\begin{array}{l}\text { ATCTGGCACCACACCTTCTA } \\
\text { CGTCATACTCCTGCTTGCTG }\end{array}$ & 835 & 62 & 35 \\
\hline
\end{tabular}

aAll sequences are mentioned from the $5^{\prime}$ to $3^{\prime}$ direction. ' In base pairs. 'Annealing temperature. 
electrophoresed on $2 \%$ agarose gels and visualised by ethidium bromide staining. All primers for RT - PCR spanned at least two exons to avoid contamination by genomic DNA. Expression of the house-keeping gene (actin) was examined at the same experimental conditions for each kallikrein, but the gel was run separately.

To verify the identity of the PCR products, they were cloned into the pCR 2.1-TOPO vector (Invitrogen, Carlsbad, CA, USA) according to the manufacturer's instructions. The inserts were sequenced from both directions using vector-specific primers, with an automated DNA sequencer. Each experiment was confirmed at least twice and the results were shown to be consistent.

\section{RESULTS}

\section{Serial analysis of gene experssion and VNB for kallikrein} expression in breast cancer

As shown in Table 3, seven kallikreins were found to be downregulated in breast cancer compared to normal breast tissues and cell lines. Probing all breast libraries from different sources (cancerous and normal tissues and cell lines) with KLK5-specific probes revealed that while $K L K 5$ was detectable in five out of eight normal breast libraries with moderate-to-high density (average expression of $319 \mathrm{tpm}$ ), mRNA tags were detectable in only one out of 24 cancer libraries. This library is a 'carcinoma in situ' and showed $34 \mathrm{tpm}$ expression level. KLK6-specifc tags were also detectable in three out of eight normal libraries with high density (average $270 \mathrm{tpm}$ ) and in only two cancer libraries (out of 24) with low average expression level $(15 \mathrm{tpm})$. KLK8 showed a generally lower expression in normal breast (average $27 \mathrm{tpm}$ ). However, expression was undetectable in the 24 cancer libraries screened. Moderate KLK10 expression levels (average $85 \mathrm{tpm}$ ) were detected in four out of eight normal breast tissues, but expression was undetectable in all cancer libraries tested. Slightly higher expression levels were detected for KLK11 and KLK12 in normal breast compared to cancer, although not statistically conclusive (Table 3 ). It is worth mentioning, however, that the only cancer library where $K L K 11$ was detected was described in the database as 'Low grade malignancy, with some in situ component'. The results for KLK3 were inconclusive, as low expression was detected in only one normal library, but not in cancer libraries (Table 3). A low number of KLK1 and KLK13 mRNA messages were detectable in the MCF7 breast cancer cell line $3 \mathrm{~h}$ after oestradiol stimulation ( $16 \mathrm{tpm}$ for each gene), but not in unstimulated cells (data not shown). No significant change of expression levels was detected in the ZR-75

Table 3 In silico analysis of kallikrein gene expression in normal and breast cancer tissues and cell lines screened by SAGE database

\begin{tabular}{|c|c|c|c|}
\hline Kallikrein & Library & Positivity (\%) & Average density $^{a}$ \\
\hline \multirow[t]{2}{*}{ KLK3 } & Normal breast & 1/8 (13) & 26 \\
\hline & Breast cancer & $0 / 24(0)$ & 0 \\
\hline \multirow[t]{2}{*}{ KLK5 } & Normal breast & $5 / 8(62)$ & 319 \\
\hline & Breast cancer & $1 / 24(4)$ & 34 \\
\hline \multirow[t]{2}{*}{ KLK6 } & Normal breast & $3 / 8(37)$ & 270 \\
\hline & Breast cancer & $2 / 24(8)$ & 15 \\
\hline \multirow[t]{2}{*}{$K L K 8$} & Normal breast & $2 / 8(25)$ & 27 \\
\hline & Breast cancer & $0 / 24(0)$ & 0 \\
\hline \multirow[t]{2}{*}{ KLKIO } & Normal breast & $4 / 8(50)$ & 85 \\
\hline & Breast cancer & $0 / 24(0)$ & 0 \\
\hline \multirow[t]{2}{*}{$K L K I I$} & Normal breast & $3 / 8(37)$ & 40 \\
\hline & Breast cancer & $1 / 24(4)$ & 28 \\
\hline \multirow{2}{*}{$K L K / 2$} & Normal breast & $1 / 8(13)$ & 15 \\
\hline & Breast cancer & $0 / 24(0)$ & 0 \\
\hline
\end{tabular}

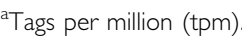

breast cancer cell line when stimulated by oestrogen (data not shown).

\section{Analysis of kallikrein gene expression utilising EST databases}

Our EST library screening results (Table 4) were consistent with SAGE expression and VNB data. While very few EST clones were retrieved from the 14 EST cancer libraries (nine for KLK8, two for $K L K 3$, and one for each of the rest), EST clones were much more detectable in normal libraries. In all, 25 KLK5 EST clones were detected in three out of eight normal breast libraries. KLK6 showed higher expression levels; 32 EST clones were found in five out of eight libraries screened. Totally, 25 KLK10 EST clones were detected in five out of eight normal libraries, and 14 clones were positive for KLK8 in two out of eight normal libraries. Two EST clones for $K L K 3$ were detected in breast cancer. It should be emphasised, however, that EST figures are more useful as 'qualitative' data. Quantitative EST results are only approximate and cannot be relied upon, due to the fact that some EST libraries are normalised. No other kallikreins were found to be differentially expressed in breast cancer using SAGE or EST databases.

\section{$\mathrm{X}$-profiler and DDD analysis of kallikrein gene expression in the breast}

Table 5 shows the analysis of kallikrein gene expression in normal and cancerous breast tissues utilising the 'X-profiler' analysis tool. A pool of eight normal breast tissues and cell lines was compared to a second pool of 24 breast cancer tissues and cell lines

Table 4 In silico analysis of kallikrein gene expression in normal and cancerous breast tissues and cell lines based on the EST databases

\begin{tabular}{|c|c|c|c|}
\hline Kallikrein & Library type & Positivity & $\begin{array}{l}\text { Number } \\
\text { of clones }\end{array}$ \\
\hline \multirow[t]{2}{*}{ KLK3 } & Normal breast & $0 / 7$ & 0 \\
\hline & Breast cancer & $1 / 14$ & 2 \\
\hline \multirow[t]{2}{*}{ KLK5 } & Normal breast & $3 / 8$ & 25 \\
\hline & Breast cancer & $1 / 14$ & । \\
\hline \multirow[t]{2}{*}{ KLK6 } & Normal breast & $5 / 8$ & 32 \\
\hline & Breast cancer & $1 / 14$ & I \\
\hline \multirow[t]{2}{*}{$K L K 8$} & Normal breast & $2 / 8$ & 14 \\
\hline & Breast cancer & $1 / 14$ & 9 \\
\hline \multirow[t]{2}{*}{ KLKIO } & Normal breast & $5 / 8$ & 25 \\
\hline & Breast cancer & $1 / 14$ & । \\
\hline \multirow[t]{2}{*}{$K L K I I$} & Normal breast & $2 / 8$ & 10 \\
\hline & Breast cancer & $0 / 14$ & 0 \\
\hline \multirow[t]{2}{*}{$K L K / 2$} & Normal breast & $1 / 8$ & 2 \\
\hline & Breast cancer & $0 / 14$ & 0 \\
\hline
\end{tabular}

Table 5 X-Profiler analysis of kallikrein gene expression in normal and cancerous breast tissues and cell lines

\begin{tabular}{lccc}
\hline & \multicolumn{2}{c}{ Gene expression counts } & \\
\cline { 2 - 3 } Kallikrein & Cancer $^{\mathbf{a}}$ & Normal $^{\mathbf{b}}$ & \\
\hline KLK5 & 2 & 7 & Difference factor \\
C & \\
KLK6 & 2 & 6 & 0.797 \\
$K L K 10$ & 0 & 6 & 0.716 \\
KLKI2 & 2 & 4 & 0.877 \\
\hline
\end{tabular}

${ }^{a}$ A pool of 22 breast cancer SAGE libraries. ${ }^{b}$ A pool of seven normal breast SAGE libraries. 'A factor of one represents the highest statistical significance. 
(all non-normalised). Our results clearly indicate significant differences in the expression levels of four kallikreins in breast cancer compared to normal. KLK5, 6, 10 and 12 were all downregulated in cancer compared to normal. The most significant result was obtained for KLK10 (difference factor of $0.877)$.

No statistically significant differences were detected by the DDD, except for $K L K 3$, where a 10 -fold increase was found in cancer when comparing the pool of normal and breast cancer libraries.

\section{Evaluation of the in silico analysis}

We verified the reliability of the in silico analysis with previously published reports on kallikrein expression in normal tissues (Yoshida et al, 1998; Yousef and Diamandis, 2000, 2003; Yousef et al, 2001, 2000b, 2000d). These results were in general agreement with PCR and Northern blotting data (Gan et al, 2000; Clements et al, 2001; Yousef and Diamandis, 2001). For example, our SAGE and EST data indicate that KLK2-4 are highly expressed in the prostate, while $K L K 5$ is highly expressed in normal breast tissue and skin, in agreement with published experimental reports (Brattsand and Egelrud, 1999; Yousef and Diamandis, 1999). In addition, we found that those kallikreins that show differential expression in breast cancer have similar levels of expression in normal and cancer tissues in other malignancies (data not shown), further verifying the specificity of our results.

\section{RT - PCR analyses in normal and cancerous breast tissues}

The expression patterns of two kallikreins were further verified for $K L K 5,6,8,10,12$ and 15 by RT-PCR analysis in histologically confirmed normal $(N=4)$ and cancerous $(N=14)$ breast tissues. As shown in Figure 1, while KLK5 was expressed at high levels in all four normal breast tissues, it was only detectable in three out of 14 cancerous breast tissues examined. KLK6 was strongly positive in normal breast tissue, but was not expressed in nine out of 14 tumour tissues, lower than normal in three and compared to normal in two (data not shown). KLK8 also showed a strong band in normal tissues, compared to undetectable expression in five tumour tissues, lower than normal in seven tumours and compared to or more than normal in two (Figure 2A). KLK10 showed no expression in eight tumours, lower than normal in five and compared to normal in one tumour (Figure 2). Figure 3 shows that while KLK12 was detectable in three out of four normal breast tissues, it was expressed at relatively lower levels in six out of 14 cancers. We also analysed KLK15 expression (as a negative control with no expression difference by in silico analysis), and found no difference in expression between normal and breast tumour tissues (data not shown).

\section{DISCUSSION}

Our results indicate that at least four kallikrein genes are downregulated in breast cancer. These data are consistent with earlier reports indicating differential expression of kallikreins in breast cancer (Diamandis and Yousef, 2001; Diamandis and Yousef, 2002; Yousef and Diamandis, 2002a). The KLK10 gene (previously known as normal epithelial cell-specific gene 1, NES1) was originally cloned by subtractive hybridisation based on its downregulation during oncogenic transformation of human mammary epithelial cells (Liu et al, 1996). More recently, it was suggested to have tumour suppressor activity (Goyal et al, 1998). Using a highly sensitive and specific immunoassay, we found hK10 expression in normal breast and in milk of lactating women (Luo et al, 2001b). hK10 was also found to be a potential marker for other malignancies, like testicular and ovarian cancers (Luo et al, 2001a, c, 2003). Recently, Band and colleagues have shown, using in situ hybridisation, lower levels of KLK10 expression in breast cancer cells when compared to normal cells (Dhar et al, 2001).

Our results with different tools (SAGE, EST and X-profiler) were consistent for KLK5, 6 and 10. The results for KLK3, however, were not clear cut. Whereas the SAGE data showed a slight downregulation in cancer compared to normal, the EST and DDD results indicate an overexpression in breast cancer. Neither of the above results was robust enough to draw a conclusion. The SAGE data were obtained from only one library and the level of expression was 'low'. The EST data were also only two clones from one library. In addition, some of the libraries used for DDD were normalised or subtracted, thus reducing the quantitative meaning of the results. This apparent discrepancy might be due to the overall low expression levels of $K L K 3$ in the breast, leading to undetectable transcripts in many libraries. Earlier, it was reported that $K L K 3$ (PSA) is not prostate specific and that it is also expressed in many other tissues including the female breast, albeit at much lower concentrations (Black and Diamandis, 2000). The potential prognostic importance of hK3 expression in breast cancer has also been reported (Yu et al, 1995).

Significant differences in expression levels were obtained for $K L K 12$, especially when comparing the normal and cancer pools (Table 5). These results follow the general pattern of downregulation of kallikreins in breast cancer. Our previous results indicate moderate KLK11 mRNA expression in the normal mammary gland (Yousef et al, 2000c). We have also previously shown that KLK5 mRNA is highly expressed in normal breast tissue (Yousef and Diamandis, 1999) and found potential prognostic significance of KLK5 expression in breast cancer (Yousef et al, 2002; 2003).

The KLK6 gene (previously known as zyme, protease $\mathrm{M}$, neurosin, PRSS6) was originally cloned based on its lower expression in metastatic breast cancer compared to primary cancer and normal tissue (Anisowicz et al, 1996). In this study, the mRNA was detectable in one out of two normal cell lines, two out of three primary breast cancer and one out of nine metastatic breast cancer cell lines. Considering the small sample number in the previous study, it was difficult to draw a definite conclusion about the expression of this gene in different phases of cancer.

Nacht et al have recently used a novel approach of combining SAGE analysis with array technology to find genes differentially expressed in breast cancer (Nacht et al, 1999). Comparing one normal cell line $v s$ two primary tumour cell lines, they identified $K L K 6$ as one of the differentially expressed genes between normal and primary breast cancer (a 2.4-fold increase in cancer). It is possible that these results reflect an individualised behaviour of certain cancer cell line. Our results have the advantage of comparing pools of normal and cancerous tissues, rather than individual cell lines. In a more recent analysis by Seth et al,

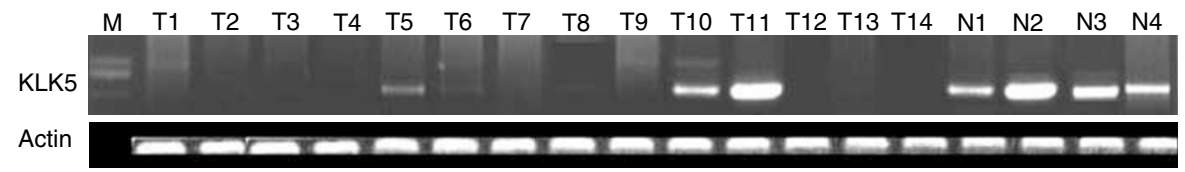

Figure I Expression of the $K L K 5$ gene in breast tumour ( $\mathrm{TI}-\mathrm{T} \mid 4)$ and normal (NI-N4) tissues. While high-expression levels were detectable in al normal tissues, the gene was expressed in only three out of I 4 cancerous tissues. Actin, a house-keeping gene, was used as a control. M, molecular weight marker. 
A

\begin{tabular}{lllllllllll}
$\mathrm{M}$ & $\mathrm{N}$ & $\mathrm{T} 1$ & $\mathrm{~T} 2$ & $\mathrm{~T} 3$ & $\mathrm{~T} 4$ & T5 & T6 & T7 & T8 & Neg. \\
\hline & & & & & & & & &
\end{tabular}

B

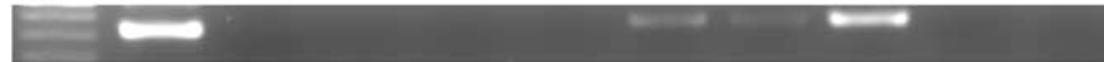

Figure 2 Representative gels showing differential expression of: $(\mathbf{A}) K L K 8,(\mathbf{B}) K L K 10$, in normal $(\mathrm{N})$ and tumour $(\mathrm{T})$ breast tissues. For discussion, see text. M, molecular weight marker.

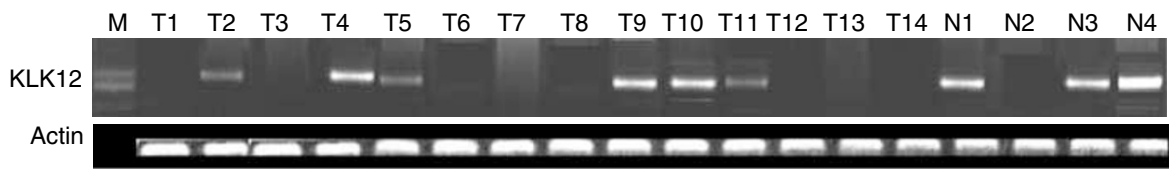

Figure 3 Expression of the KLKI2 gene in breast tumour $(\mathrm{TI}-\mathrm{TI} 4)$ and normal $(\mathrm{NI}-\mathrm{N} 4)$ tissues. Actin, a house-keeping gene, was used as a control. M, molecular weight marker.

kallikreins 5 and 6 were identified among 35 transcripts that were most abundantly expressed in oestrogen receptor (ER)-positive normal human breast tissue (Seth et al, 2002). Interestingly, comparing their constructed SAGE library tags with other normal, in situ, invasive and metastatic carcinoma tags from different SAGE libraries, their data indicate that these two kallikreins are exclusively expressed in normal breast tissues (Seth et al, 2002).

The hK6 protein was recently shown to be a potential marker for ovarian cancer (Diamandis et al, 2003). Also, in a recent analysis, the median serum concentration of hK6 was found to be higher in normal females (median expression of $7.0 \mu \mathrm{gl}^{-1}$ ) compared to breast cancer patients (median expression of $4.3 \mu \mathrm{gl}^{-1}$ ) (Diamandis et al, 2000a). In addition, the hK6 protein was found at high concentrations in breast tissue, milk of lactating women, nipple aspirate fluid and breast cyst fluid, with lower levels detectable in breast tumour cytosols (Diamandis et al, 2000b).

A noteworthy observation is the presence of a group of closely localised kallikreins (KLK5, 6, 8, 10, 11 and 14) with a similar pattern of differential expression in breast cancer. This points to the possibility of the existence of a locus control region or another common regulatory mechanism that controls the parallel expression of these kallikreins.

Other kallikrein genes, for example, KLK13 and KLK14, were also previously reported to be downregulated in breast cancer (Yousef et al, 2000a, b, 2001). Our analyses did not show any detectable expression of these genes by either SAGE or EST methods. This is likely due to the fact that these genes are expressed in the breast at very low levels that are not detectable, except by sensitive techniques like RT - PCR.

It should be emphasised that although in silico analysis is a useful research method, the results should be verified by more than one analytical tool, and further experimentally confirmed. Possible sources of bias include sequence errors, presence of specific sequence mutations associated with certain malignancies,

\section{REFERENCES}

Adams MD, Soares MB, Kerlavage AR, Fields C, Venter JC (1993) Rapid cDNA sequencing (expressed sequence tags) from a directionally cloned human infant brain cDNA library. Nat Genet 4: 373-380

Anisowicz A, Sotiropoulou G, Stenman G, Mok SC, Sager R (1996) A novel protease homolog differentially expressed in breast and ovarian cancer. Mol Med 2: 624-636

Barry MJ (2001) Clinical practice. Prostate-specific-antigen testing for early diagnosis of prostate cancer. N Engl J Med 344: 1373-1377

Benson DA, Karsch-Mizrachi I, Lipman DJ, Ostell J, Rapp BA, Wheeler DL

(2002) GenBank. Nucleic Acids Res 30: 17-20 unequal presentation of different physiological or pathological libraries and the expression of splice variants in certain malignancies.

Lercher et al, during global analysis of over 11000 genes in 14 different tissues, have set a cut-off value for gene expression as being low $(\leqslant 37 \mathrm{tpm})$ and intermediate or high $(\geqslant 134 \mathrm{tpm})$ (Lercher et al, 2002). If this cut-off is to be applied to our data, we can conclude that while kallikrein gene expression is absent or low in breast cancer, medium-to-high levels of expression are found in the normal breast.

Several hypotheses can be proposed regarding the possible mechanism(s) by which kallikreins may be involved in cancer. KLK3 was previously shown to have an antiangiogenic and a tumour suppressor effect on the growth of some breast cancer cell lines (Lai et al, 1996; Fortier et al, 1999). Breast cancer is a 'hormonal' malignancy. Kallikreins, being under steroid hormone regulation, may represent downstream targets by which hormones influence the initiation or progression of cancer. Another possible mechanism for the involvement of kallikreins in malignancy is the activation of proteinase-activated receptors (PAR). The activation of these receptors elicits different responses in several tissues.

It is important to mention that mRNA levels might not be necessarily be associated with a decrease in the serum or tissue levels of these proteins. As is the case with hK3, the serum rise might be due to 'leakage' from malignant cells due to architectural and angiogenic changes. We have recently shown elevation of hK5 levels in the serum of some patients with breast cancer (Yousef et al, 2003).

In conclusion, we provide strong evidence suggesting that at least four kallikreins are downregulated in breast cancer. These data were confirmed from several databases and by experimental analysis with RT - PCR. It will be useful to examine these kallikreins as biomarkers for diagnosis, prognosis and treatment decisions in breast cancer.

Black MH, Diamandis EP (2000) The diagnostic and prognostic utility of prostate-specific antigen for diseases of the breast. Breast Cancer Res Treat 59: $1-14$

Brattsand M, Egelrud T (1999) Purification, molecular cloning, and expression of a human stratum corneum trypsin-like serine protease with possible function in desquamation. J Biol Chem 274: $30033-30040$

Clements J, Hooper J, Dong Y, Harvey T (2001) The expanded human kallikrein (KLK) gene family: genomic organisation, tissue-specific expression and potential functions. Biol Chem 382: 5-14 
Dhar S, Bhargava R, Yunes M, Li B, Goyal J, Naber SP, Wazer DE, Band V (2001) Analysis of normal epithelial cell specific-1 (NES1)/Kallikrein 10 mRNA expression by in situ hybridization, a novel marker for breast cancer. Clin Cancer Res 7: 3393-3398

Diamandis EP, Scorilas A, Fracchioli S, Van Gramberen M, De Bruijn H, Henrik A, Soosaipillai A, Grass L, Yousef GM, Stenman UH, Massobrio M, Van Der Zee AG, Vergote I, Katsaros D (2003) Human kallikrein 6 (hK6): a new potential serum biomarker for diagnosis and prognosis of ovarian carcinoma. J Clin Oncol 21: 1035-1043

Diamandis EP, Yousef GM (2001) Human tissue kallikrein gene family: a rich source of novel disease biomarkers. Expert Rev Mol Diagn 1: $182-190$

Diamandis EP, Yousef GM (2002) Human tissue kallikreins: a family of new cancer biomarkers. Clin Chem 48: 1198-1205

Diamandis EP, Yousef GM, Soosaipillai AR, Bunting P (2000a) Human kallikrein 6 (zyme/protease M/neurosin): a new serum biomarker of ovarian carcinoma. Clin Biochem 33: 579-583

Diamandis EP, Yousef GM, Soosaipillai AR, Grass L, Porter A, Little S, Sotiropoulou G (2000b) Immunofluorometric assay of human kallikrein 6 (zyme/protease M/neurosin) and preliminary clinical applications. Clin Biochem 33: $369-375$

Fortier AH, Nelson BJ, Grella DK, Holaday JW (1999) Antiangiogenic activity of prostate-specific antigen. J Natl Cancer Inst 91: 1635-1640

Gan L, Lee I, Smith R, Argonza-Barrett R, Lei H, McCuaig J, Moss P, Paeper B, Wang K (2000) Sequencing and expression analysis of the serine protease gene cluster located in chromosome 19q13 region. Gene 257: 119-130

Goyal J, Smith KM, Cowan JM, Wazer DE, Lee SW, Band V (1998) The role for NES1 serine protease as a novel tumor suppressor. Cancer Res 58: $4782-4786$

Lai LC, Erbas H, Lennard TW, Peaston RT (1996) Prostate-specific antigen in breast cyst fluid: possible role of prostate-specific antigen in hormonedependent breast cancer. Int J Cancer 66: 743 - 746

Lercher MJ, Urrutia AO, Hurst LD (2002) Clustering of housekeeping genes provides a unified model of gene order in the human genome. Nat Genet 31: $180-183$

Liu XL, Wazer DE, Watanabe K, Band V (1996) Identification of a novel serine protease-like gene, the expression of which is down-regulated during breast cancer progression. Cancer Res 56: 3371-3379

Luo L, Bunting P, Scorilas A, Diamandis EP (2001a) Human kallikrein 10: a novel tumor marker for ovarian carcinoma? Clin Chim Acta 306: $111-118$

Luo LY, Grass L, Howarth DJ, Thibault P, Ong H, Diamandis EP (2001b) Immunofluorometric assay of human kallikrein 10 and its identification in biological fluids and tissues. Clin Chem 47: 237-246

Luo LY, Katsaros D, Scorilas A, Fracchioli S, Bellino R, Van Gramberen M, De Bruijn H, Henrik A, Stenman UH, Massobrio M, Van Der Zee AG, Vergote I, Diamandis EP (2003) The serum concentration of human kallikrein 10 represents a novel biomarker for ovarian cancer diagnosis and prognosis. Cancer Res 63: 807-811

Luo LY, Rajpert-De Meyts ER, Jung K, Diamandis EP (2001c) Expression of the normal epithelial cell-specific 1 (NES1; KLK10) candidate tumour suppressor gene in normal and malignant testicular tissue. $\mathrm{Br} \mathrm{J}$ Cancer 85: $220-224$

Nacht M, Ferguson AT, Zhang W, Petroziello JM, Cook BP, Gao YH, Maguire S, Riley D, Coppola G, Landes GM, Madden SL, Sukumar S (1999) Combining serial analysis of gene expression and array technologies to identify genes differentially expressed in breast cancer. Cancer Res 59: $5464-5470$

Seth P, Porter D, Lahti-Domenici J, Geng Y, Richardson A, Polyak K (2002) Cellular and molecular targets of estrogen in normal human breast tissue. Cancer Res 62: $4540-4544$
Strausberg RL (2001) The Cancer Genome Anatomy Project: new resources for reading the molecular signatures of cancer. J Pathol 195: 31-40

Strausberg RL, Greenhut SF, Grouse LH, Schaefer CF, Buetow KH (2001) In silico analysis of cancer through the Cancer Genome Anatomy Project. Trends Cell Biol 11: S66-S71

Tanimoto H, Underwood LJ, Shigemasa K, Yan Yan MS, Clarke J, Parmley TH, O'Brien TJ (1999) The stratum corneum chymotryptic enzyme that mediates shedding and desquamation of skin cells is highly overexpressed in ovarian tumor cells. Cancer 86: 2074-2082

Velculescu VE, Zhang L, Vogelstein B, Kinzler KW (1995) Serial analysis of gene expression. Science 270: 484-487

Yoshida S, Taniguchi M, Suemoto T, Oka T, He X, Shiosaka S (1998) cDNA cloning and expression of a novel serine protease, TLSP. Biochim Biophys Acta 1399: $225-228$

Yousef GM, Chang A, Diamandis EP (2000a) Identification and characterization of KLK-L4, a new kallikrein-like gene that appears to be downregulated in breast cancer tissues. J Biol Chem 275: $11891-11898$

Yousef GM, Diamandis EP (1999) The new kallikrein-like gene, KLK-L2 Molecular characterization, mapping, tissue expression, and hormonal regulation. J Biol Chem 274: 37511-37516

Yousef GM, Diamandis EP (2000) The expanded human kallikrein gene family: locus characterization and molecular cloning of a new member, KLK-L3 (KLK9). Genomics 65: 184-194

Yousef GM, Diamandis EP (2001) The new human tissue kallikrein gene family: structure, function, and association to disease. Endocr Rev 22: $184-204$

Yousef GM, Diamandis EP (2002a) Expanded human tissue kallikrein family - a novel panel of cancer biomarkers. Tumour Biol 23: 185-192

Yousef GM, Diamandis EP (2002b) Human tissue kallikreins: a new enzymatic cascade pathway? Biol Chem 383: 1045-1057

Yousef GM, Diamandis EP (2003) Human kallikreins: common structural features, sequence analysis and evolution. Curr Genom 4: 147-165

Yousef GM, Magklara A, Chang A, Jung K, Katsaros D, Diamandis EP (2001) Cloning of a new member of the human kallikrein gene family, KLK14, which is down-regulated in different malignancies. Cancer Res 61: $3425-3431$

Yousef GM, Magklara A, Diamandis EP (2000b) KLK12 is a novel serine protease and a new member of the human kallikrein gene familydifferential expression in breast cancer. Genomics 69: 331-341

Yousef GM, Polymeris ME, Grass L, Soosaipillai A, Chan PC, Scorilas A, Borgono C, Harbeck N, Schmalfeldt B, Dorn J, Schmitt M, Diamandis EP (2003) Human kallikrein 5: a potential novel serum biomarker for breast and ovarian cancer. Cancer Res 63: 3958-3965

Yousef GM, Scorilas A, Diamandis EP (2000c) Genomic organization, mapping, tissue expression, and hormonal regulation of trypsin-like serine protease (TLSP PRSS20), a new member of the human kallikrein gene family. Genomics 63: 88-96

Yousef GM, Scorilas A, Kyriakopoulou LG, Rendl L, Diamandis M, Ponzone R, Biglia N, Giai M, Roagna R, Sismondi P, Diamandis EP (2002) Human kallikrein gene 5 (KLK5) expression by quantitative PCR: an independent indicator of poor prognosis in breast cancer. Clin Chem 48: $1241-1250$

Yousef GM, Scorilas A, Magklara A, Soosaipillai A, Diamandis EP (2000d) The KLK7 (PRSS6) gene, encoding for the stratum corneum chymotryptic enzyme is a new member of the human kallikrein gene family genomic characterization, mapping, tissue expression and hormonal regulation. Gene 254: 119-128

Yu H, Giai M, Diamandis EP, Katsaros D, Sutherland DJ, Levesque MA, Roagna R, Ponzone R, Sismondi P (1995) Prostate-specific antigen is a new favorable prognostic indicator for women with breast cancer. Cancer Res 55: $2104-2110$ 